\title{
Padrão epidemiológico da esquistossomose em comunidade de pequenos produtores rurais de Pernambuco, Brasil 1
}

\author{
Schistosomiasis epidemiological patterns in a \\ community of small farmers in Pernambuco State, \\ Brazil 1
}

Constança Simões Barbosa 2

Frederico Simões Barbosa 2

\footnotetext{
1 Trabalho financiado pelo Programa de Pequeñas Becas, Laboratório de Ciencias Sociales, Universidad Central deVenezuela - TDR/WHO.

2 Centro de Pesquisas Aggeu Magalhães, Fundação Oswaldo Cruz.

Av. Moraes Rego s/no, Recife, PE 520020-020, Brasil.
}

Abstract This article is part of a larger study in which epidemiological and anthropological methods were used to hel $p$ understand the production and maintenance of schistosomiasis in a small endemic area in Pernambuco State. A cross-sectional study identified several local risk factors for schistosomiasis, quantifying socioeconomic, sanitary, and behavioral variables and then relating them to the prevalence and intensity of schistosomiasis infection. Using uni variate analysis, three variables (age group, schooling, and human/water contact) showed significant association with S. mansoni infection. Causal factors and confounding variables were identified through multivariate analysis. Quantitative epidemiological analysis is critically discussed re garding a qualitative ethnographic study in relation to environmental risk situations (contamination and transmission) as well as risk practices (economic and behavioral).

Key words Schistosomiasis; Public Health; Risk Factors; Epidemiology

Resumo Este artigo é parte de um estudo mais amplo que empregou abordagens epidemi ológica eantropológica para procurar entender a produção e manutenção da esquistossomose em pe quena comunidade de área endêmica de Pernambuco. No presente trabalho, realizamos um estudo de corte transversal para avaliação dos fatores de risco para esquistossomose, associando variáveis sócio-econômicas, sanitárias e comportamentais (quantificáveis), com a prevalência e intensi dade da infecção. Uma análise estatística univariada selecionou três variáveis (faixa etária, escolaridade e número de contatos), que mostraram associação significativa com a positi vidade para S. mansoni. Os resultados da análise multivariada realizada a seguir permitiram a identificação dos fatores causais e das variáveis de confundimento, entre aquel as anteriormente sel eci onadas. O diagnóstico epi demiológico quantitati vo é discutido criticamente, com embasamento no estudo quali tativo etnográfico, quanto às condições ambi entais de ri sco (contaminação, transmissão) e práticas de risco (ativi dades econômicas e comportamentais). Palavras-chave Esquistossomose; Saúde Pública; Fatores de Risco; Epidemiologia 


\section{Introdução}

O presente trabalho discute al guns aspectos da epidemiologia da esquistossomose em Natuba, localidade en dêmica da zona rural de Pernambuco. É parte de um estudo mais amplo que pretendeu fazer a articulação do conhecimento epidemiológico com o antropológico no sentido de compreender uma situação endêmica nas suas várias dimensões e níveis determinantes, objetivando identificar elementos relevantes para a construção de ações programáticas de controle em nível local (Barbosa, 1996).

As situações de transmissão da esquistossomose em Pernambuco são tão variadas, como são a ecologia e os grupos sociais nos quais ela ocorre. A prevalência e a intensidade da infecção nas comunidades afetadas pela doença estão sujeitas a variações relacionadas a práticas culturalmente moldadas pelas atividades econômicas, de lazer ou domésticas, que são peculiares em cada localidade. As medidas de controle deveriam contemplar estas diversas esferas, se almejam lograr eficiência nas suas ações.

Dados do órgão oficial de controle de endemias, Fundação Nacional de Saúde (FNS), revelam que Pernambuco é o segundo colocado em números de casos positivos (para o Nordeste), com prevalência média de 15,2\% (Kano, 1992). No entanto, este indicador de morbidade não retrata situações epidemiológicas específicas, como, por exemplo, localidades com $80 \%$ de prevalência (Barbosa \& Silva, 1992), que mereceriam atenção especial.

Levantamento realizado nos arquivos da FNS permitiu-nos conhecer a evolução das prevalências para a esquistossomose em Natuba, por intermédio dos inquéritos coproscópicos ali realizados. Em 1977, a prevalência foi de $37,9 \%$, tendo havido, em 1978, tratamento em massa. Em 1980, os boletins registram 19,1\% de prevalência, não tendo sido encontrado nenhum registro de tratamento. Em 1993, a taxa é de $44,3 \%$ e neste mesmo ano houve tratamento seletivo. Vale salientar que, durante a década de 80, as epidemias emergentes no Estado concentraram toda a mão-de-obra técnica disponível na FNS, havendo uma desmobilização do Programa de Esquistossomose. Este perfil da evolução das taxas de prevalência com tendência à estabilidade é típico das localidades rurais de Pernambuco, sendo um reflexo da ineficácia das medidas imediatistas de controle (quimioterapia e aplicação de moluscicida) e do desconhecimento acerca da multiplicidade de causas que mantém a endemia.
Os fatores responsáveis pela manutenção e reprodução destas taxas de prevalência em Pernambuco já foram devidamente discutidos (Barbosa et al., 1996).

As considerações acima justificam nosso interesse em proceder a um estudo epidemiológico, de corte transversal, para conhecer os padrões biológicos, sociais, econômicos, sanitários e comportamentais de risco, associá-los à prevalência e intensidade da infecção para avaliar a expressão da morbidade da esquistossomose na localidade em estudo. Como resultado deste trabalho específico, teremos um diagnóstico sobre o padrão epidemiológico local, que, aliado a outros conhecimentos resgatados por meio de estudos paralelos, congregará subsídios para a elaboração de estratégias mais coerentes para o controle da endemia.

\section{Área de estudo}

As terras da antiga fazenda Natuba, situada no Município de Vitória de Santo Antão, distam cerca de $65 \mathrm{~km}$ de Recife, capital do Estado de Pernambuco. Natuba está situada na região fisiograficamente conhecida como Zona da Mata, originariamente coberta por vegetação típica de floresta (Mata Atlântica). O desmatamento desta região ocorreu em razão do estabelecimento de extensos latifúndios de cana-de-açúcar que avançaram para o interior do Estado.

A necessidade de expansão destes latifúndios, ditada pela introdução das destilarias de álcool, marcou também a expropriação dos pequenos produtores das terras ocupadas por eles (Heredia, 1989). Em al gumas raras localidades da Zona da Mata de Pernambuco, como Natuba e Galiléia, antigos trabalhadores dos engenhos conseguiram legalmente a posse da terra em que vinham trabalhando há décadas como cortadores de cana e ali se estabeleceram, em sistema de cooperativas, para produção agrícola mais diversificada.

O clima da região é quente e úmido, com uma estação de chuvas que vai de maio a agosto. A localidade possui 117 casas com 511 moradores, segundo censo demográfico realizado pela nossa equipe em 1994. A maioria das residências é de alvenaria com banheiros e/ ou fossas.

A forma de ocupação da terra é caracterizada pelos minifúndios, repartidos eqüitativamente entre as famílias produtoras. A base da produção econômica dos moradores está centrada no cultivo de hortaliças.

O único recurso hídrico natural da região é o rio Canha, nas margens do qual foram insta- 
ladas bombas de sucção para alimentar o sistema de irrigação por aspersão manual, por meio de mangueiras distribuídas pelas hortas. Algumas práticas usadas para a agricultura, como o represamento do rio em alguns pontos para a lavagem das verduras e o bombeamento da água para as hortas, propiciam criadouros artificiais para os moluscos transmissores da esquistossomose que aí se reproduzem em abundância.

A comunidade possui uma escola municipal de primeiro grau e um pequeno posto de saúde com uma atendente e dois agentes comunitários, mantidos pela prefeitura. O posto conta com atendimento médico duas vezes por semana e os agentes de saúde são membros da comunidade. Possuem uma estrutura políticocomunitária surpreendentemente bem organizada para os padrões regionais, com uma Associação dos Pequenos Agricultores de Natuba.

Levantamentos realizados nos boletins de coproscopia nos arquivos da FNS revelaram que a prevalência para esquistossomose em Natuba vem se mantendo estável há décadas (30\% a 40\%), apesar das sucessivas intervenções do programa oficial de controle.

Metodologia de campo e de laboratório

O trabal ho de campo para coleta de dados foi realizado entre os meses de janeiro a dezembro de 1994.

Trabalhando com toda a população, foi realizado um levantamento seccional objetivando determinar a prevalência e a intensidade de esquistossomose na localidade. Buscou-se identificar os fatores de risco, associando as variáveis sócio-econômicas, sanitárias e comportamentais quantificáveis com a prevalência e intensidade da infecção, mediante cálculo da estimativa do risco relativo pelo odds-ratio.

Nos meses de janeiro a fevereiro, elaborouse um croqui da localidade onde foram demarcadas as residências, vias de acesso transitáveis e coleções d'água. As residências foram cadastradas, recebendo número e registro próprios. De março a agosto foi realizado o inquérito demográfico, sócio-econômico, sanitário e o estudo comportamental de risco, em todas as 117 famílias da localidade, utilizando-se, como instrumento de coleta, questionário previamente testado.

A coleta das amostras de fezes para análise parasitológica foi realizada nos meses de agosto a outubro em toda a população de Natuba, mediante a distribuição de recipientes numerados, recolhidos em prazo máximo de 24 ho- ras. O material foi processado pelo método de sedimentação simples e Kato-Katz, para pesquisa de helmintos e protozoários, tendo sido examinadas duas lâminas para cada indivíduo.

Logo após o levantamento e mapeamento das coleções hídricas, foi feita uma coleta preliminar de moluscos em todos os ambientes aquáticos, pelo método de Olivier $\&$ Schneiderman (1956), para caracterizar a espécie transmissora e sua ocorrência nos diversos criadouros. Os caramujos coletados foram conduzidos ao laboratório para a identificação da espécie e verificação de positividade para Schistosoma mansoni pelo teste de eliminação de cercárias. As estações de coleta foram definidas levandose em consideração não só o espaçamento geográfico entre os criadouros, como também aqueles pontos onde a população tinha contatos freqüentes. As coletas de moluscos foram realizadas uma vez por mês, no período de novembro de 1993 a outubro de 1994.

\section{A nálise dos dados}

Para efeito da determinação dos fatores de risco, práticas e situações específicas de risco, foram selecionadas as seguintes variáveis:

- Dependentes: prevalência e intensidade da infecção.

- Independentes: sexo, idade, inserção no sistema produtivo, nível de escolaridade, renda mensal da família, origem, tempo de residência na área, freqüência do contato com águas naturais, motivo do contato com águas naturais, distância da casa para os focos, origem da água para uso doméstico, instalações sanitárias, destino do conteúdo das fossas.

Os dados foram estocados em banco de dados D base e analisados estatisticamente pelo SAS (Statistcs Anal isys System), determinandose os testes de significância e análise multivariada (odds ratio - OR - com 95\% de intervalo de confiança - IC) para testar a força de associação entre as variáveis independentes e a prevalência e intensidade de infecção esquistossomótica.

\section{Resultados e comentários}

Inquérito demográfico, sócio-econômico, sanitário e comportamental de risco

Natuba conta atualmente com 117 residências e 511 moradores. Em cerca de $55 \%$ dos domicílios vivem famíllias constituídas por três a cinco indivíduos. A maior parte dos moradores $(58,9 \%)$ reside na localidade há mais de seis 
anos e 81,8\% são originários do próprio município.

Quanto ao modo de inserção no sistema produtivo, $66,7 \%$ das famílias são pequenos proprietários rurais e agricultores e outros 19,7\% também trabalham na terra como agregados ou empregados.

Embora 47,5\% dos 446 moradores entrevistados sejam analfabetos, o percentual de pessoas com primeiro grau completo $(28,3 \%)$ é elevado para a área rural de Pernambuco, considerando-se os índices regionais (FIBGE, 1992). O mesmo acontece com a renda familiar das 117 famílias entrevistadas, que se concentra na faixa de dois salários mínimos (50,4\%), e com o tipo de construção, sendo $90,6 \%$ das casas de alvenaria. Estes indicadores sócio-econômicos dão uma idéia da diferença da qualidade de vida de pequenos proprietários rurais em uma região onde predominam os latifúndios, caso em que esses índices ostentam valores bem inferiores.

A maioria das residências (69,3\%) está situada a mais de $100 \mathrm{~m}$ de distância do rio, no entanto 85,3\% dos moradores têm contato diário e intenso com as águas deste pelo tipo de atividade agrícola da comunidade, não sendo relevante, neste caso, o fator de proximidade da casa ao foco.

O inquérito sanitário informa que 90,6\% das casas possuem instalações para o destino dos dejetos (privada efossa). A maioria das fossas, quando enche, é esvaziada no peridomicílio, $(59,9 \%)$ o que condiciona o risco de contaminação, pois o declive dos terrenos facilita o carreamento das fezes para o rio, por ocasião das chuvas.

Quanto ao tipo de contato dos habitantes com as águas naturais (rio), as atividades de risco predominantes são banhos $(86,4 \%)$ e práticas agrícolas (63,2\%). Quanto à origem e ao uso das águas utilizadas pela família, cerca de $80,0 \%$ delas utilizam o rio para atividades de trabalho, higiene e lazer.

\section{Inquérito coproscópico}

Ao todo foram distribuídos 516 recipientes na comunidade. Destes, 450 foram recolhidos e 439 permitiram a realização de exame parasitológico. O percentual de recusas e de indivíduos não encontrados foi de $14,9 \%$. As Tabelas 1 e 2 indicam, respectivamente, a prevalência das parasitoses intestinais e a intensidade da infecção por S. mansoni.

$O$ resultado dos exames de fezes mostra a prevalência das parasitoses encontradas (Tabela 1) e a intensidade da infecção por S. mansoni (Tabela 2).

\section{Inquérito malacológico}

A única espécie de caramujo de interesse epidemiológico foi a Biomphalaria straminea, com 8.489 exemplares coletados. Foram examinados 7.065 exemplares, todos com resultado negativo para eliminação de cercárias de $S$. mansoni. A mortalidade dos caramujos em laboratório, até a época do tercei ro exame, foi de $16,8 \%$. A maior concentração de caramujos por criadouro foi verificada na estação seca do ano.

\section{A nálise estatística dos resultados}

O uso do teste Qui-Quadrado de Associação elegeu algumas das variáveis independentes que mostraram associação significativa com a positividade para a infecção humana por S. mansoni. Estas variáveis foram: faixa etária, nível de escolaridade e número de contatos com a água. A variável número de contatos foi agrupada em três categorias: nenhum contato, um ou dois contatos e três ou mais contatos.

Os resultados da análise multivariada (tendo como base as variáveis independentes acima mencionadas) e a relação entre essas variáveis com a prevalência e cargas parasitárias da infecção pelo S. mansoni estão nas Tabelas 3 a 6 .

As variáveis faixa etária e número de contatos foram analisadas conjuntamente para verificação de uma relação estatística entre elas e a probabilidade de infecção para S. mansoni.

Riscos estatisticamente significativos para a infecção $(O R=1,58)$ foram obtidos para a faixa de 10-39 anos com mais de três contatos com os focos de transmissão. Há correlação positiva entre aumento do número de contatos, em cada uma das faixas etárias e risco de infecção.

As variáveis nível de escolaridade e número de contatos foram analisadas conjuntamente para verificação de uma relação estatística entre elas e a probabilidade de infecção para S. mansoni.

Riscos estatisticamente significativos para a infecção $(O R=1,88)$ foram obtidos para a categoria ler/ escrever, com mais de três contatos com os focos de transmissão. Novamente foi encontrada correlação positiva entre aumento do número de contatos e risco de infecção, independentemente do nível de instrução. A variável número de contatos foi significativa no nível de significância considerado no ajuste do modelo, enquanto a variável nível de escolaridade não foi significativa, sendo as probabilidades de significância de 0,001 e 0,1682 respectivamente. $\mathrm{O}$ modelo foi bem aceito com razão 
de verossimilhança igual a 2,11 e probabilidade de significância de 0,7762.

As cargas parasitárias dos indivíduos positivos para S. mansoni foram correlacionadas com as variáveis faixa etária e número de contatos para verificação de relação estatística entre estas e a intensidade de infecção.

Para qualquer faixa etária, se não foi mencionado contato com coleções hídricas, a probabilidade de carga parasitária foi aproximadamente zero.

Considerando-se os graus de infecção de um a três e o número de contatos não nulos, a faixa etária de dez a 39 anos tem todas as probabilidades iguais ou superiores à faixa etária de zero a nove anos. A soma das probabilidades para os graus de um a três foi superior para os indivíduos que tiveram três ou mais contatos, independente da faixa etária.
Tabela 1

Prevalência das parasitoses intestinais em 439 residentes de Natuba, Município de Vitória de Santo Antão, Pernambuco, julho de 1994.

\begin{tabular}{lcc}
\hline Espécies & $\begin{array}{c}\text { Método Kato-Katz } \\
\%\end{array}$ & $\begin{array}{c}\text { Método Hofmann } \\
\%\end{array}$ \\
\hline Ascaris lumbricoides & 8,6 & 7,2 \\
Ancilostomídeos & 2,8 & 24,9 \\
Trichuris trichiura & 7,9 & 6,3 \\
Enterobius vermicularis & 0,5 & 1,6 \\
Hymenolepis nana & 0,5 & 4,9 \\
Strongiloydes stercoralis & 0,2 & 6,3 \\
Endolimax nana & - & 3,9 \\
Entamoeba coli & 0,5 & 16,1 \\
Entamoeba histolytica & - & 3,7 \\
Giardia lamblia & - & 4,9 \\
Schistosoma mansoni & 35,1 & 17,2 \\
\hline
\end{tabular}

\section{Tabela 2}

Intensidade da infecção por Schistosoma mansoni, por faixa etária, em 145 casos positivos de residentes em Natuba, Município de Vitória de Santo Antão, PE, julho de 1994.

\begin{tabular}{|c|c|c|c|c|c|c|}
\hline \multirow{2}{*}{$\begin{array}{l}\text { Número de ovos/ } \\
\text { grama de fezes }\end{array}$} & \multicolumn{5}{|c|}{ Faixa etária (\%) } & \multirow[t]{2}{*}{ Total (\%) } \\
\hline & 0 a 6 & 7 a 14 & 15 a 25 & 26 a 40 & $>40$ & \\
\hline 11 a 100 & 3,5 & 14,5 & 13,8 & 11,7 & 10,3 & 53,8 \\
\hline 101 a 400 & 2,1 & 10,3 & 6,9 & 5,5 & 7,6 & 32,4 \\
\hline mais de 400 & - & 3,4 & 5,5 & 2,8 & 2,1 & 13,8 \\
\hline Total & 5,6 & 28,2 & 26,2 & 20,0 & 20,0 & 100,0 \\
\hline
\end{tabular}

Tabela 3

Distribuição dos casos de esquistossomose em residentes de Natuba, Município de Vitória de Santo Antão, Pernambuco, segundo a faixa etária e número de contatos com águas naturais.

\begin{tabular}{|c|c|c|c|c|c|c|c|}
\hline Faixa etária & $\begin{array}{l}\mathrm{N} \text { úmero } \\
\text { de contatos }\end{array}$ & Positivos & $(\%)$ & $\begin{array}{l}\text { Número de } \\
\text { examinados }\end{array}$ & $\begin{array}{l}\text { Probabilidade } \\
\text { estimada }\end{array}$ & Odds ratio & IC $(95 \%)$ \\
\hline \multirow[t]{3}{*}{0 a 9} & 0 & 0 & - & 9 & 0 & - & - \\
\hline & 1 ou 2 & 10 & 2,3 & 85 & 0,1399 & 0,1627 & $0,0997-0,2658$ \\
\hline & 3 ou mais & 20 & 4,5 & 39 & 0,4641 & 0,8660 & $0,5275-1,4219$ \\
\hline \multirow[t]{2}{*}{10 a 39} & 0 & 0 & - & 6 & 0 & - & _ \\
\hline & 3 ou mais & 72 & 16,4 & 121 & 0,6122 & 1,5792 & $1,1307-2,2056$ \\
\hline \multirow[t]{3}{*}{40 ou mais } & 0 & 0 & - & 9 & 0 & - & - \\
\hline & 1 ou 2 & 10 & 2,3 & 49 & 0,2079 & 0,2624 & $0,1536-0,4483$ \\
\hline & 3 ou mais & 20 & 4,6 & 34 & 0,5827 & 1,3964 & $0,8193-2,3801$ \\
\hline Total & & 154 & 35,1 & 439 & - & - & - \\
\hline
\end{tabular}




\section{Tabela 4}

Distribuição dos casos de Schistosoma mansoni em residentes de Natuba, Município de Vitória de Santo Antão, Pernambuco, segundo o nível de escolaridade e número de contatos com águas naturais.

\begin{tabular}{|c|c|c|c|c|c|c|c|}
\hline $\begin{array}{l}\text { Nível de } \\
\text { escolaridade }\end{array}$ & $\begin{array}{l}\text { Número } \\
\text { de contatos }\end{array}$ & Positivos & $\%$ & Total & $\begin{array}{l}\text { Probabilidade } \\
\text { estimada }\end{array}$ & Odds ratio & IC $(95 \%)$ \\
\hline \multirow[t]{3}{*}{ Analfabetos } & 0 & 0 & - & 15 & 0 & - & - \\
\hline & 1 ou 2 & 22 & 5,0 & 127 & 0,1745 & 0,2114 & $0,1426-0,3133$ \\
\hline & 3 ou mais & 41 & 9,3 & 76 & 0,5373 & 1,1613 & $0,7868-1,7141$ \\
\hline \multirow[t]{3}{*}{ ler/escrever } & 0 & 0 & - & 5 & 0 & - & - \\
\hline & 1 ou 2 & 8 & 1,8 & 40 & 0,2571 & 0,3425 & $0,2058-0,5702$ \\
\hline & 3 ou mais & 44 & 10,0 & 64 & 0,6530 & 1,8820 & $1,2069-2,9348$ \\
\hline \multirow[t]{3}{*}{ lo grau } & 0 & 0 & - & 4 & 0 & - & - \\
\hline & 1 ou 2 & 12 & 2,7 & 54 & 0,1738 & 0,2170 & $0,1304-0,3611$ \\
\hline & 3 ou mais & 27 & 6,1 & 54 & 0,5438 & 1,1924 & $0,7558-1,8810$ \\
\hline Total & & 154 & 35,0 & 439 & - & - & - \\
\hline
\end{tabular}

Tabela 5

Carga parasitária para infecção por Schistosoma mansoni, em residentes de $\mathrm{N}$ atuba, Município de Vitória de Santo Antão, Pernambuco, segundo faixa etária e número de contatos com águas naturais.

\begin{tabular}{|c|c|c|c|c|c|c|}
\hline \multirow{2}{*}{$\begin{array}{l}\text { Faixa etária } \\
\text { (anos) }\end{array}$} & \multirow{2}{*}{$\begin{array}{l}\text { Número } \\
\text { de contatos }\end{array}$} & \multicolumn{4}{|c|}{ Carga parasitária* (probabilidade estimada) } & \multirow{2}{*}{$\begin{array}{l}\text { Total } \\
\text { freq. }\end{array}$} \\
\hline & & $\begin{array}{c}0 \\
\text { freq. }(\%)\end{array}$ & $\begin{array}{c}1 \\
\text { freq. }(\%)\end{array}$ & $\begin{array}{c}2 \\
\text { freq. }(\%)\end{array}$ & $\begin{array}{c}3 \\
\text { freq. }(\%)\end{array}$ & \\
\hline \multirow[t]{3}{*}{0 a 9} & nenhum & $9(0,9999)$ & 0 & 0 & 0 & 9 \\
\hline & 1 ou 2 & $75(0,8650)$ & $8(0,1039)$ & $2(0,0310)$ & 0 & 85 \\
\hline & 3 ou mais & $19(0,5248)$ & $3(0,0555)$ & $11(0,2657)$ & $6(0,1536)$ & 39 \\
\hline \multirow[t]{3}{*}{10 a 39} & nenhum & $6(0,9999)$ & 0 & 0 & 0 & 6 \\
\hline & lou 2 & $65(0,7537)$ & $19(0,2090)$ & $3(0,0372)$ & 0 & 87 \\
\hline & 3 ou mais & $49(0,4001)$ & $11(0,0976)$ & $34(0,2790)$ & $27(0,2231)$ & 121 \\
\hline \multirow[t]{3}{*}{40 ou mais } & nenhum & $9(0,9999)$ & 0 & 0 & 0 & 9 \\
\hline & 1 ou 2 & $39(0,8141)$ & $7(0,1424)$ & $3(0,0434)$ & 0 & 49 \\
\hline & 3 ou mais & $14(0,3855)$ & $2(0,0593)$ & $9(0,2903)$ & $9(0,2647)$ & 34 \\
\hline Total & & 285 & 50 & 62 & 42 & 439 \\
\hline
\end{tabular}

* cargas parasitárias: $1=0$ a 100 opg; $2=101$ a 300 opg; 3 = mais de 300 opg.

As cargas parasitárias dos indivíduos positivos para S. mansoni foram correlacionadas com as variáveis nível de escolaridade e número de contatos para verificação de relação estatística entre estas e a intensidade de infecção. A variável número de contatos foi significativa, ao contrário da variável nível de escolaridade, com as probabilidades de significância de 0,6603 e 0,0001 respectivamente.

Esse modelo logístico foi excelentemente bem aceito, com razão de verossimilhança igual a 2,87 e probabilidade de significância de 0,9964 .
Para qualquer nível de escolaridade, se não houve referência a contato com águas naturais, a probabilidade de infecção é aproximadamente zero.

Considerando-se as cargas parasitárias de um a três e o número de contatos não nulos, os que sabem ler e escrever têm todas as probabilidades maiores do que as correspondentes das outras categorias de nível de escolaridade.

A soma das probabilidades para as cargas parasitárias é superior para os indivíduos que mantiveram três ou mais contatos, independente do nível de escolaridade. 
Distribuição dos casos de Schistosoma mansoni, por carga parasitária, em residentes de Natuba, Município de Vitória de Santo Antão, Pernambuco, segundo nível de escolaridade e número de contatos com coleções hídricas.

\begin{tabular}{|c|c|c|c|c|c|c|}
\hline \multirow{2}{*}{$\begin{array}{l}\text { Nível de } \\
\text { escolaridade }\end{array}$} & \multirow{2}{*}{$\begin{array}{l}\text { Número } \\
\text { de contatos }\end{array}$} & \multicolumn{4}{|c|}{ Carga parasitária* (probabilidade estimada) } & \multirow{2}{*}{$\begin{array}{l}\text { Total } \\
\text { freq. }\end{array}$} \\
\hline & & $\begin{array}{c}0 \\
\text { freq. }(\%)\end{array}$ & $\begin{array}{c}1 \\
\text { freq. }(\%)\end{array}$ & $\begin{array}{c}2 \\
\text { freq. } \%\end{array}$ & $\begin{array}{c}3 \\
\text { freq. }(\%)\end{array}$ & \\
\hline \multirow[t]{3}{*}{ Analfabetos } & nenhum & $15(0,9999)$ & 0 & 0 & 0 & 15 \\
\hline & 1 ou 2 & $105(0,8278)$ & $17(0,1372)$ & $5(0,0348)$ & 0 & 127 \\
\hline & 3 ou mais & $35(0,4586)$ & $6(0,0732)$ & $20(0,2707)$ & $15(0,1973)$ & 76 \\
\hline \multirow[t]{3}{*}{ ler/escrever } & nenhum & $5(0,9999)$ & 0 & 0 & 0 & 5 \\
\hline & 1 ou 2 & $32(0,7457)$ & $7(0,2039)$ & $1(0,0503)$ & 0 & 40 \\
\hline & 3 ou mais & $20(0,3464)$ & $7(0,0912)$ & $22(0,3279)$ & $15(0,2343)$ & 64 \\
\hline \multirow[t]{3}{*}{$10 \mathrm{grau}$} & nenhum & $4(0,9999)$ & 0 & 0 & 0 & 4 \\
\hline & 1 ou 2 & $42(0,8153)$ & $10(0,1556)$ & $2(0,0289)$ & 0 & 54 \\
\hline & 3 ou mais & $27(0,4624)$ & $3(0,0850)$ & $12(0,2303)$ & $12(0,2222)$ & 54 \\
\hline Total & & 285 & 50 & 62 & 42 & 439 \\
\hline
\end{tabular}

* cargas parasitárias: 1 = 0 a 100 opg; $2=101$ a 300 opg; 3 = mais de 300 opg.

\section{Discussão e conclusões}

As taxas de prevalência para esquistossomose, na população de Natuba, vêm se mantendo estáveis há décadas. Ao contrário do que ocorre entre os cortadores de cana-de-açúcar da região, a exposição ao risco (contatos com a água) é sistemática para quase todos os residentes nesta localidade. A atividade econômica que praticam condiciona o uso contínuo das águas contaminadas e 85,3\% dos moradores têm contatos diários prolongados. Diante deste quadro de sistemática exposição ao risco, era de se esperar uma alta prevalência para esquistossomose, assim como elevadas cargas parasitárias. Contudo, os resultados mostram $35,1 \%$ de prevalência e 53,8\% da população com carga parasitária leve.

Quando estas mesmas variáveis foram analisadas conjuntamente (análise multivariada) com a variável número de contatos com a água do rio, houve sempre uma correlação positiva entre o aumento no números de contatos que as pessoas tinham com as águas e o risco de infecção, independente dos níveis das faixas etárias e escolaridade. Pelos resultados desta análise, podemos considerar a variável número de contatos como fator causal, enquanto a faixa etária e escolaridade estariam atuando como variáveis de confusão.

Estes fatores de risco e suas correlações são reconhecidos por vários estudos epidemiológicos em esquistossomose, como os de Lima e Costa et al. (1987), Barreto (1993), Ximenes
(1991) e Coura-Filho et al. (1994), entre outros. Os resultados obtidos pelo cálculo do odds rati o são compatíveis com o conhecimento que se tem sobre contatos com águas contaminadas por cercárias de S. mansoni como fator de risco para a infecção (Guimarães et al., 1985; Loureiro, 1978; Barreto, 1993). Contudo, a confirmação destes padrões, em Natuba, mostrouse insuficiente para uma compreensão holística da realidade endêmica, onde fatores causais não quantificáveis também se expressam.

Observações resultantes da vivência no dia-a-dia com a comunidade permitiram uma avaliação qualitativa das condições de risco específicas. Alguns aspectos mereceram destaque como componentes e coadjuvantes da estrutura epidemiológica local.

Em Natuba, a contaminação fecal dos criadouros de moluscos é baixa e não permanente. Como a maioria das casas possui fossas $(90,6 \%)$, as fezes não são depositadas diretamente no rio. Algumas delas apresentavam vazamentos, cujo conteúdo ressecava no próprio quintal. Nestes casos, os ovos deS. mansoni só conseguiam alcançar as águas quando eram arrastados pelas chuvas até o rio. Outra situação de risco, que condiciona contaminação fecal esporádica do ambiente, está no fato de que algumas fossas necessitam ser esvaziadas uma vez ao ano, sendo seu conteúdo lançado em terrenos adjacentes ao quintal das casas, podendo ser carreado, na estação das chuvas, até o rio ou às canaletas de irrigação das hortas. 
O modo de produção agrícola e as condições ambientais foram, ao longo do tempo, moldando as práticas coletivas para otimização da produtividade, que acabaram por se transformar em fatores de risco adicionais para a transmissão da esquistossomose. Por exemplo, o sistema de irrigação adotado leva caramujos e cercárias para dentro das hortas e a lavagem das verduras no rio condiciona os agricultores a uma exposição contínua.

Confrontamos, a seguir, a situação epidemiológica (prevalência x carga parasitária x exposição x contaminação fecal) com os resultados do inquérito malacológico que determinou a variação mensal da densidade dos moluscos transmissores e a taxa de infecção dos mesmos. Não foi encontrado nenhum caramujo infectado pelo S. mansoni entre os 7.065 exemplares submetidos a três exames cada, em períodos diferentes. Este resultado não significa a ausência de contaminação da água por cercárias, pois B. straminea é responsável por altas taxas de endemicidade em situações nas quais as pesquisas malacológicas para positividade à infecção dão negativas (Barbosa \& Silva, 1992).

Uma outra observação pode estar relacionada com os resultados negativos dos exames dos caramujos e, conseqüentemente, com as baixas taxas de infecção humana exibidas, considerando a exposição contínua dos moradores. Durante todo o ano em que permanecemos em contato diário com a comunidade, não detectamos, em nenhuma ocasião, pessoas defecando ao ar livre, próximo ao rio ou em outros locais, hábito este muito comum em residentes da zona rural canavieira. Provavelmente isto se deve ao fato de que as famílias, além de possuírem banheiros em suas casas, trabaIham muito próximo das mesmas, podendo facilmente satisfazerem suas necessidades fisiológicas com privacidade.

O estudo antropológico realizado na localidade (Barbosa, 1996) identificou as crenças e percepções da população em relação à etiologia e transmissão da endemia. Por exemplo, as sanguessugas, presentes em grande quantidade na lama das hortas, são percebidas como exercendo um papel preponderante como vetor da doença. São elas, e não os caramujos, que exercem uma ação espoliativa direta, podendo, ao sugar o sangue, infectar as pessoas.

A análise estatística dos fatores de risco selecionou variáveis e determinou a probabilidade de os indivíduos adquirirem a esquistossomose, mas a epidemiologia quantitativa possui limitações quanto à explicação causal dos fenômenos, associando, simplesmente, características individuais e doença, não levando em conta especificidades locais nem a natureza histórica e sócio-cultural dos eventos somáticos. Conseqüentemente, não produz conhecimento suficiente para orientar medidas de saúde pública comprometidas com a modificação da qualidade de vida de grupos sociais menos favorecidos.

Fatores de risco são mais que taxas construídas segundo variáveis coletadas no meio ambiente. Eles se expressam também na rotina diária das populações que vivem em uma região endêmica, cujas práticas e crenças somente podem ser compreendidas quando seus 'mundos' são explorados (Agar, 1996). Não podemos esquecer ainda que, quando o comportamento individual é reduzido a aspectos quantificáveis de conduta, está-se enfatizando a necessidade de controle individual, social e moral (Merchán-Hamann, 1995).

O estudo dos fatores de risco foi o referencial metodológico que nos permitiu diagnosticar instantaneamente as variáveis independentes que pudessem estar associadas à transmissão da esquistossomose na localidade. A partir deste perfil epidemiológico, o estudo etnográfico nos direcionou para o conhecimento e o entendimento das relações sociais e culturais que permeiam estas variáveis para que estas passassem a adquirir sentido e coerência diante da realidade endêmica de Natuba (Barbosa, 1996). A opção de compreender uma realidade endêmica, utilizando estes dois métodos de análise, proporcionou-nos uma concepção holística e mais humana do processo, uma vez que o conhecimento dos elementos condicionantes do adoecer observados, quantificados, ou resgatados na subjetividade do imagi nário popular, foi ampliado, complementado e enriquecido.

O modelo integrado para análise de uma situação endêmica (Barbosa, 1996), construído com base nestes conhecimentos, mostrou-se capaz de gerar um nível de entendimento dos problemas de saúde de pequenas comunidades a partir do qual é possível apontar na direção das medidas sociais e sanitárias necessárias ao controle da transmissão da esquistossomose. Além disto, a viabilidade prática da execução metodológica deste tipo de estudo capacita sua aplicação em projetos descentralizados de controle de endemias, enriquecendo a análise e discussão na busca de soluções conjuntas entre técnicos e comunidade. 


\section{Agradecimentos}

Aos técnicos e colegas da Estação de Campo do Programa de Esquistossomose do CpqAM, pela valiosa colaboração nas etapas de laboratório e campo; ao Dr. Ubiracy Guida, Coordenador do Programa de Controle de Endemias da Fundação Nacional de Saúde/PE, pelo apoio em todas as fases deste trabal ho e ao Prof. José Edmilson Mazza, do Dep. de Estatística da UFPE, pela análise estatística dos resultados.

\section{Referências}

AGAR, M., 1996. Recasting the "Ethno" in "Epidemiolgy". Medical Anthropology, 16:391-403.

BARBOSA, C. S., 1996. Esquistossomose em Pernambuco: Determinantes Bio-Ecológi cos e Sócio-Culturais em Comunidade de Pequenos Agricultores da Zona da Mata. Tese de Doutorado, Rio de Janeiro: Escola Nacional de Saúde Pública, Fundação Oswaldo Cruz.

BARBOSA, C. S.; SILVA, C. B. \& BARBOSA, F. S., 1996 Esquistossomose em Pernambuco: reprodução e expansão da endemia. Revista de Saúde Pública, 30:609-616.

BARBOSA, C. S. \& SI LVA, C. B., 1992. Epidemiologia da esquistossomose mansônica no Engenho Bela Rosa, Município de São Lourenço da Mata, Pernambuco. Cadernos de Saúde Pública, 8:83-87.

BARRETO, M. L., 1993. Use of risk factors obtained by questionary in the screening for Schistosomiasis infection. American Journal of Tropical Medicine and Hygiene, 48:742-744.

COURA-FILHO, P.; ROCHA, R. S.; FARAH, M. W.; SILVA, G. C. \& KATZ, N., 1994. Identification of risk groups for infection with Schistosoma mansoni: a strategy for implementation of control measures? Revista do Instituto de Medicina Tropical de São Paulo, 36:245-253.

FIBGE (Fundação Instituto Brasileiro de Geografia e Estatística), 1992. Censo Demográfico (Dados Distritais). Vol. 1, tomo 3 (10), Rio de Janeiro: FIBGE.

GUIMARÃES, M. D. C.; LIMA E COSTA, M. F. F.; LIMA, L. B. \& MOREIRA, M. A., 1985. Estudo clínico-epidemiológico da esquistossomose mansônica em escolares do Município de Arcos, Minas Gerais. Revista deSaúde Pública, 19:8-17.
HEREDIA, B. M. A., 1989. Formas de Dominação e Espaço Social: A Modernização da Agroindústria Canavieira em Alagoas. São Paulo/Brasília: Marco Zero/CNPq.

KANO, P. H., 1992. Medidas para o controle da esquistossomose adotadas pela Fundação Nacional de Saúde. Memórias do Instituto Oswaldo Cruz, 87: 315-320.

LIMA E COSTA, M. F. F.; MAGALHÃES, M. H. A.; ROCHA, R. S.; ANTUNES, C. M. F. \& KATZ, N. 1987. Water contact patterns and socio-economic variables in the epidemiology of schistosomiasis mansoni in endemic area in Brazil. Bulletin of the World Health Organization, 65:57-66.

LOUREIRO, S., 1978. Schistosomiasis Mansoni in Children: An Epidemiological Study of Patterns of Water Exposure Using Path-Analysis. PhD Thesis, Houston: School of Public Health, University of Texas.

MERCHÁN-HAM AN, E., 1995. Grau de informação, atitudes e representações sobre o risco e prevenção da AIDS em adolescentes do Rio de Janeiro, Brasil. Cadernos de Saúde Pública, 11:463-478.

OLIVIER, L. \& SCHNEIDERMAN, M., 1956. A method for estimating the density of aquatic snail populations. Experimental Parasitology, 5:109-117.

XIMENES, R. A. A., 1991. Urbanization of Schistosomiasis in a Metropolitan Area of Recife, Brazil. Social Causation of Schistosomiasis in theAge Group 10-Years in the Urban Area of São Lourenço da Mata. PhD Thesis, London: London School of Hygiene and Tropical Medicine, University of London. 